\title{
The Survival Processing Memory Effect Should Generalise to Source Memory, but It Doesn't
}

\author{
Arndt Bröder ${ }^{1}$, Nelli Krüger ${ }^{2}$, Susanne Schütte ${ }^{2}$ \\ ${ }^{1}$ Chair of Experimental Psychology, University of Mannheim, Mannheim, Germany; \\ ${ }^{2}$ Department of Psychology, University of Bonn, Bonn, Germany. \\ Email: broeder@uni-mannheim.de \\ Received September 29 $9^{\text {th }}, 2011$; revised October 29 $9^{\text {th }}, 2011$; accepted November $29^{\text {th }}, 2011$.
}

\begin{abstract}
Numerous studies have shown that words judged for their relvance to a scenario of survival are remembered better than words from lists processed differently. Survival processing is even more effective than many mnemonic techniques. This has been interpreted as an evolutionary design feature of memory. It is argued that such a survival effect should be even more pronounced in remembering the context or source of the information. Two experiments used a source monitoring paradigm. Both studies did not find any evidence for enhanced context memory, thus questioning the evolutionary account of the survival processing effect. An alternative interpretation is discussed.
\end{abstract}

Keywords: Survival Processing, Source Memory, Evolutionary Psychology

\section{Introduction}

Since its first demonstration by Nairne, Thompson and Pandeirada (2007), the "survival processing effect" in memory has stimulated a great deal of research. In the basic paradigm, participants of an experimental group judge the relevance of a list of words with respect to a "survival scenario" in which they imagine that they were stranded on a foreign grassland without supplies, and they have to survive for several weeks. In a typical control condition, the list of words is rated with respect to another scenario without the survival topic, or they process the words in various other ways (see Naine \& Pandeirada, 2008, for an overview). The experimental condition usually evokes much better memory in a surprise recall or recognition test than most of the control conditions tested until now, even if those use efficient mnemonic techniques (e.g., pleasantness ratings, imagery, item generation, self-reference ratings, or intentional learning; Nairne, Pandeirada, \& Thompson, 2008) or other interesting or arousing scenarios as a control (Kang, McDermott, \& Cohen, 2008; Nairne et al., 2008; Weinstein, Bugg, \& Roediger, 2008).

The discoverers of this powerful survival processing advantage seek an explanation in evolutionary terms: "We propose that memory evolved to enhance reproductive fitness and, accordingly, its systems are tuned to retain information that is fitness-relevant." (Nairne \& Pandeirada, 2008). Further, the authors claim that a functional analysis based on evolutionary principles is lacking in most cognitive domains, possibly overlooking other highly relevant variables that determine cognitive functioning.

Several authors claimed that other confounded proximal factors might trigger the effect, such as the congruity of words to the scenario (Butler, Kang, \& Roediger, 2009), the distinctiveness of item processing (Kroneisen \& Erdfelder, 2011) or the emotional arousal produced by survival processing (see e.g., Soderstrom \& McCabe, 2011, for effects with "zombie" scenarios). Nairne and colleagues have countered those criticisms by claiming that survival effects remain if congruity is controlled (Nairne \& Pandeirada, 2011). Also, more exciting alter- native scenarios (Kang et al., 2008) or scenarios with similar amounts of distinctiveness (Nairne, Pandeirada, \& Thompson, 2008) produced the effect. The purpose of this paper is not to present another possible confound or mediator of the effect. Rather, we test a corollary of the adaptive memory hypothesis. To be of functional importance, we claim that remembering items per se is not enough. Rather, the context has to be remembered in which these items appeared since the context often determines the item's relevance. This hypothesis was already stated in a similar fashion by Nairne et al. (2007, p. 264).

In the following, we will first argue that a survival processing effect should be found in source memory. Then, we present two experiments in which the source (spatial location) of items was varied in addition to the scenario of the rating task. Third we will discuss what the failure to find a survival processing advantage in source memory means for the evolutionary account put forward by Nairne and Pandeirada (2008).

\section{Source Memory}

The term "source" refers to the temporal and spatial circumstances under which a memory was acquired as well as to other features present in the encoding situation (such as internal states of or cognitive processes; see Johnson, Hashtroudi, \& Lindsay, 1993). This broad definition is therefore roughly equivalent to the entire context of learning an item or a piece of information. In formal theories of memory, episodic memories are descibed as feature vectors which entail the item information as well as some context information (Flexser \& Tulving, 1978). Contextual features can therefore act as powerful retrieval cues for the target information (Godden \& Baddeley, 1975). The capacity for source memory is partly dissociable from item memory: It suffers more than item memory in amne-

\footnotetext{
${ }^{1}$ After we had conducted our experiments, we learned that Nairne et al (2010) had investigated a similar hypothesis with apparently similar procedures and results which they presented at the meeting of the Psychonomic Society in November 2010. We will address this briefly in the General Discussion.
} 
sia (Shimamura \& Squire, 1987) or age-related memory decline (Spencer \& Raz, 1995). Also, experimental manipulations can affect item memory and source memory idependently (Bayen, Murnane, \& Erdfelder, 1996; Meiser \& Bröder, 2002). In their seminal paper, Johnson et al. (1993) already emphasized that source monitoring is a complex interaction of retrieval and inference processes rather than a mere passive retrieval.

Why should source memory be relavant for an evolutionary account of memory? Although Naine and Pandeirada (2008) claim that their survival processing effect is compatible with a functional interpretation, the exact nature of the utility of enhanced memory for specific items is somewhat elusive. What functional relevance per se does it have to rember that I encountered a "saw" or a "lion" in a survival-relevant scenario? In which way does this in itself benefit survival or reproduction? In our view, remembering the item is only helpful if and only if I also rember the context in which I encountered it. It is important to know where the waterhole is and when deer usualy graze near the forest. Also, I should know who provided valuable information and where I can find sticks and stones for making tools. It is of no particular adaptive value to know that all these items were encountered, but it is very helpful if the context of these encounters is remembered. Hence, we predict that the survival processing effect must also be found in source memory for items if the evolutionary explanation is correct. Otherwise, the functional analysis remains incomplete.

In two experiments, we tested this corollary of the adaptive memory account by employing the usual scenario-based procedure with the additional variation of the context in which items appeared. Later, item memory and context memory were assessed in a recognition test.

\section{Experiment 1}

The aim of this experiment was to replicate the survival processing effect in item memory and to assess the impact of survival processing on source memory for the location of the items.

\section{Design}

Three factors were varied. Items in the final source memory test were either old or new. Old words had been rated before for their relevance with respect to one of two different scenarios: the foreign grassland scenario (survival processing) or the fancy vacation resort scenario (no survival processing). This factor was manipulated by different instructions which were translated to German from Nairne et al. (2007). The vacation scenario was used as a control condition in order to roughly equate richness and distinctiveness of processing. Finally, the location on the screen during the learning phase was varied within participants. The words appeared in one of 16 squares that were arranged in a large 4 by 4 square on the computer screen.

\section{Materials and Procedure}

The 128 words were taken from Nairne et al. (2007; Exp. 3) and translated to German. They were randomly divided into two sets of 64 each, one of which was the learning set, the other was the distractor set for all participants, and both were shuffled in a random order for the relevance ratings. Each of the 64 learning list words was randomly assigned to one of the 16 presentation locations for each participant in such a way that four words appeared in each position.

Participants were told that they should rate the relevance of a list of words with respect to a scenario described later. They were familiarized with the rating scale ranging from 1 "completely irrelevant" to 5 "highly relevant". They were asked to provide each rating within 5 seconds because all words were presented for the same amount of time. This was done in order to eliminate processing time as a potential confound. After that, participants were asked to imagine one of the two scenarios and to begin with three practice trials with additional words. After the practice trials, participants could ask for clarification and/or begin with the rating task which lasted approximately 5 minutes. Ratings were entered by clicking an appropiate numbered button at the bottom of the computer screen below the 16 squares grid in which the word appeared. A 2 minute filler task followed in which participants had to judge the result of simple calculation problems as true or false. Finally, the source memory test followed. Participants were asked to judge for 128 items whether they were old (formerly rated) or new. They had to click an appropriate button on the screen. If they clicked "new", the next word appeared. If they clicked "old", a small version of the 4 by 4 grid appeared on the screen below the word with the instruction to check the square with the former position of the word. After the 128 test words had been presented, partcipants were thanked and debriefed. The experiment lasted 17 minutes on average.

\section{Participants}

Fifty students (mostly major psychology) of the University of Bonn and employed persons participated in the study for partial course credit (psychology students) or without compensation (others). 43 participants were female, the mean age was 27 years (range 18 - 62).

\section{Results}

Although there are several caveats in using traditional source memory measures to assess source memory (Bröder \& Meiser, 2007; Murnane \& Bayen, 1996), we chose to report those here for simplicity. The structure of results is identical to those obtained with an appropriate multinomial 2-High-Threshold source memory model (Bayen et al., 1996). We will first report the results on old-new recognition, followed by source memory measures.

\section{Item Memory}

Corrected hit rates and false alarm rates according to Snodgrass and Corwin (1988) are reported in Table 1 along with the corresponding $d^{\prime}$ values for old-new recognition. The sensitivity indexes $d^{\prime}$ did not differ significantly between conditions, $t(48)$ $=.54, p=.59, d=.15$. Hence, the survival processing effect was not replicated for item recognition, the estimated effect size is small. Furthermore, this "effect" is driven entirely by somewhat smaller FARs instead of higher HRs

\section{Source Memory}

Since there is no standard procedure for measuring source memory for 16 different sources, we computed source memory in analogy to the conditional source identification measure (Murnane \& Bayen, 1996) which is based only on the items correctly identified as old and therefore does not confound item memory and source memory under regular conditions. The percentage of these items with a correct source judgment was determined for each participant. As Table 2 shows, these measures did not differ significantly between conditions, $t(48)=$ $-1.4, p=.16, \eta^{2}=.04$. The descriptive difference is in the wrong direction. To rule out a potential floor effect, one can 
Table 1.

Item memory in Experiment 1.

\begin{tabular}{cccc}
\hline & \multicolumn{3}{c}{ Item memory } \\
\cline { 2 - 4 } & Hit rate & FA rate $^{\mathrm{a}}$ & $d^{\prime}$ \\
\hline survival & $.86(.12)$ & $.06(.05)$ & $2.85(0.71)$ \\
\hline vacation & $.86(.12)$ & $.09(.05)$ & $2.73(0.85)$ \\
\hline both & $.86(.13)$ & $.08(.05)$ & $2.79(0.78)$ \\
\hline
\end{tabular}

${ }^{\mathrm{a}} \mathrm{FA}$ rate $=$ False Alarms rate

Table 2.

Source memory in Experiment 1.

\begin{tabular}{ccccc}
\hline & \multicolumn{4}{c}{ Source memory } \\
\cline { 2 - 5 } & Cell & Quadrant & Vertical & Horizontal \\
\hline Survival & $.10(.05)$ & $.34(.09)$ & $.56(.06)$ & $.63(.09)$ \\
Vacation & $.13(.07)$ & $.32(.10)$ & $.55(.08)$ & $.61(.08)$ \\
Both & $.12(.07)$ & $.33(.09)$ & $.56(.07)$ & $.62(.08)$ \\
\hline
\end{tabular}

also use more liberal source memory scoring criteria, for example whether the participant chose the correct quadrant of the display, or the correct half, either in vertical or in horizontal direction. This would signify partial context memory. Especially the horizontal location knowledge is well above chance, but there was no significant difference for any of these more liberal measures of partial source knowledge between conditions, all $t(48)<.90$, all $p>.35$, all $\eta^{2}<.02$.

\section{Discussion}

The hypothesis derived from an evolutionary functional account of the survival processing advantage received no support in this study. It was argued that survival processing should affect source memory in particular since item memory per se has hardly any survival value without appropriate context knowledge. Neither exact nor partial source knowledge were affected, however. This could not be attributed to a floor effect, at least for the partial source memory measures. Note that source memory was scored conditional on successful item memory, so this cannot be a consequence of attenuated source memory measures.

However, strict conclusions are not warranted since there was no significant effect on item memory, either. Although we used the standard procedure for inducing the survival processing effect, it may not have worked in this experiment for any unknown reason. However, the paradigm has not been used with a source memory test, yet, and this may have compromised the recognition data. Also, the effects usually tend to be weaker in recognition than in free recall. In addition, the encoding phase with 16 different source may have been too distracting, or it may have raised participants' suspicion that a memory test would follow, thus inducing potential compensatory encoding strategies.

In the second experiment, we therefore used two modifications: First, we only used two different sources which is the standard in source monitoring tasks. This should reduce distraction from the scenario instruction. Second, in order to increase the chances of finding a survival effect, we used the standard "moving scenario" as a control in the second experiment (Nairne et al., 2007). As an additional variation, we used words and pictures in this study to replicate the findings by Otgaar et al. (2010) who found the survival processing effect both for words and pictures. We expected to find a general picture superiority effect (Paivio, 1991) as well as an independent survival processing effect for both verbal and pictorial materials.

\section{Experiment 2}

In order to enhance the chances to find a survival processing effect in item memory, the standard contrast between the survival scenario and a "moving scenario" was used in which participants rate items for relevance with respect to moving to a new location. Second, pictorial and verbal items were used in a within subjects variation. Third, only two sources were used, namely the presentation of items at the top or the bottom of the screen. Also we increased sample size to achieve a higher statistical power.

\section{Materials, Design, and Procedure}

The information format (picture vs. word) was varied within participants who were tested individually. 200 simple line drawings of common objects were taken from Snodgrass and Vanderwart (1980). A parallel list of words naming these objects was created. Four counterbalanced learning lists were created so that each item appeared equally often as an old or new item, and in word or picture format across participants. In the encoding phase, each participant saw 100 items for $5 \mathrm{sec}$ onds each, 50 of which appeared in the upper half of the screen, the others in the lower half of the screen. These had to be rated on a relevance scale from 1 (irrelevant) to 5 (highly relevant) by verbalizing the numerical response which was documented by the experimenter. This was done in order to enhance participants' compliance with the task and thus to increase the chance to elicit a survival processing effect. The participants were either instructed to rate the stimuli with respect to their relevance to the typical survival scenario or the moving scenario (see Appendix). After 3 practice trials the rating task began which lasted about 8:20 Minutes. After that, simple calculation problems had to be solved on a sheet for two minutes before the final surprise source memory test was administered. This test entailed 200 items (old and new words and pictures) presented in the middle of the computer screen, and participants noted on a protocol sheet whether the item was "old top", "old bottom" or "new". Finally, the sheets were collected, and participants were thanked and debriefed.

\section{Participants}

64 persons ( 48 female, mean age $=25.94$ years, $\mathrm{SD}=5.83$ ) participated in the study. Most of them (80\%) were psychology students of the University of Bonn who received partial course credit. The others participated for candy and the chance to win a book coupon of 10 Euros value.

\section{Results}

For item memory, Hit rates and False alarm rates as well as $d^{\prime}$ values can be found in Table 3. Subjecting the d' values to a mixed model ANOVA reveals a massive materials effect, $F(1$, $62)=59.46, p<.001, \eta_{p}^{2}=.49$, showing that pictures were remembered much better than words. However, in contrast to Experiment 1, there was now also a significant scenario main effect, $F(1,62)=4.16, p<.05, \eta_{p}^{2}=.06$. Also, a significant interaction shows that the scenario effect is larger for words than pictures, $F(1,62)=5.33, p=.02, \eta_{p}^{2}=.08$. In fact, 
Table 3.

Item and source memory in Experiment 2.

\begin{tabular}{|c|c|c|c|c|c|}
\hline & & \multicolumn{3}{|c|}{ Item memory } & \multirow{2}{*}{$\begin{array}{l}\text { Source } \\
\text { memory }\end{array}$} \\
\hline & & Hit rate & FA rate ${ }^{a}$ & $d^{\prime}$ & \\
\hline \multirow{2}{*}{ survival } & $\mathrm{w}^{\mathrm{a}}$ & $.91(.09)$ & $.06(.06)$ & $3.26(0.70)$ & $.55(.09)$ \\
\hline & $p$ & $.96(.05)$ & $.05(.04)$ & $3.68(0.64)$ & $.61(.11)$ \\
\hline \multirow{2}{*}{ vacation } & $\mathrm{w}$ & $.87(.07)$ & $.08(.07)$ & $2.75(0.66)$ & $.52(.09)$ \\
\hline & $p$ & $.94(.05)$ & $.06(.06)$ & $3.47(0.77)$ & $.62(.11)$ \\
\hline \multirow{2}{*}{ both } & $\mathrm{w}$ & $.89(.08)$ & $.07(.06)$ & $3.00(0.72)$ & $.53(.09)$ \\
\hline & $p$ & $.95(.05)$ & $.06(.05)$ & $3.57(0.71)$ & $.62(.11)$ \\
\hline
\end{tabular}

${ }^{\mathrm{a}} \mathrm{w}=$ words, $p=$ pictures.

follow-up analyses show that the main effect is only significant for words, $t(62)=3.02, p=.002$, but not for pictures, $t(62)=$ $1.19, p=.12$ (one-tailed).

The number of correct source attributions conditional on successful item recognition was calculated for all participants. The source of pictures was remembered much better than the source of words, $F(1,62)=44,44, p<.001, \eta_{p}^{2}=.42$. However, there was neither a main effect of the scenario, $F(1,62)=$ $0.21, p=.65, \eta_{p}^{2}=.003$, nor an interaction of the factors, $F(1$, $62)=1.93, p=.17, \eta_{p}^{2}=.03$. Hence, in source memory, we found the expected materials effect, but again no effect of survival processing.

\section{Discussion}

In this experiment, the usual survival processing advantage could be elicited, although it did not reach significance for picture stimuli (in contrast to Otgaar et al., 2010). Hence, item memory was affected in the expected manner. However, again, there was not even a sign of an effect in source memory. The massive advantage of pictures over words in item and source memory was as expected, and it shows that large effects on these measures can be obtained. Hence, the failure to find a scenario effect cannot be explained away as a potential insensitivity of the source memory measure. The effect size estimate is close to zero.

\section{General Discussion}

The functional analysis of cognitive processes and memory can be a fruitful way to generate hypotheses about the inner workings of the mind (Anderson, 1991; Pinker, 1997). The advent of evolutionary psychology has generated a host of fruitful ideas how to understand different aspects of behavior including cognitive functions (e.g., Buss, 1999; Barrett, Dunbar, \& Lycett, 2002). However, many results that were inspired by evolutionary hypotheses may be explained by rival hypotheses (e.g. mating preferences, see the controversy between Buss, 1992 and Eagly \& Wood, 1999). Unfortunately, tests are sometimes elusive because many significant variables from the evolutionary point of view are not possible to control experimentally. This is different with Nairne's and cowokers' survival processing effect in which an experimenter-induced mode of information processing has an effect in line with an evolutionary interpretation.

However, as we have outlined in the introduction, we feel that the functional analysis in the case of the survival processing advantage is incomplete. Different from other constructs like the hypothesized "cheater detection module" (Cosmides,
1989), the functional value of merely remembering items that were encountered in a survival-relevant context remains unclear. What does it pay to know that I encountered a "chair" (or anything else) in a survival-relevant context? Even if we assume that this should be of some value, we would expect a particular advantage for relevant as opposed to irrelevant items. However, Naine and co-workers typically do not find a difference in mean relevance ratings between remembered and not-remembered items. For a more sensitive analysis, we computed the individual point-biserial correlations between the memory status of old items (miss vs. hit) and the relevance ratings given in the rating task. The mean correlations in all conditions of our experiments ranged from -.04 to .01 , none of them significantly different from zero. This independence between relevance and memory is a problematic finding for the evolutionary explanation of the survival processing advantage.

Furthermore, we argued that even more than item memory itself, source memory should be enhanced to be of functional or survival-value. Items are helpful only if they can be placed in a partciular temporo-spatial or social context. It is important where and when you will find game or fruit. Hence, if the survival processing advantage were present for item memory and source memory, a functional interpretation would immediately gain much face validity. Our results, however, do not even present a hint of such an effect in source memory, so the evolutionary explanation of the survival processing effect remains elusive. After we had conducted the experiments, we learned about similar experiments by Nairne, Pandeirada, Smith, Grimaldi, \& Bauernschmidt (2010) who also did not find survival processing advantages for source memory, however.

Of course, these two studies cannot be viewed as definitive. Although we would argue that the location of items should be their most important contextual aspect in a survival setting, our manipulation of this feature was obviously not "ecologically valid" in the sense of a resemblance to actual survival situations. On the other hand, this resemblance is also absent in judging lists of words. Nevertheless, the advantage occurs in this setting. Hence, the effect in item memory reflects some general design feature of memory which is also elicited in artificial situations. If this is true, however, we do not see why this should not be the case for context dimensions. However, perhaps more "representative" variations of context features may be used in follow-up studies.

Another apparent problem with our studies is the acceptance of the null hypothesis which is not recommended by some scientists (Wilkinson et al., 1999). The lack of a significant effect is often mistrusted. However, if the significance levels are chosen in a more liberal way to equate $\alpha$ and $\beta$ error in a compromise power analysis (assuming medium effect sizes according to Cohen, 1988), there is still no evidence for an effect. Hence, the data for all source memory measures in both studies are more likely under the null hypothesis than under the alternative hypothesis of an at least medium-sized effect. Also, the estimated effect sizes are close to zero in both studies.

In the meantime, there have been other results with the survival processing paradigm which are hard to reconcile with an evolutionary account. For example, Otgaar and Smeets (2010) as well as Howe and Derbish (2010) have found increased rates of false memories in the DRM paradigm under survival processing conditions. The fitness value of this phenomenon is at least not obvious.

In sum, our results add to some evidence which questions the evolutionary explanation of the survival processing advantage. If this trend continues, it will be worthwhile for researchers to 
address the potential proximate or mediating mechanisms which might be responsible for this powerful effect. Kroneisen (2010) has collected several candidates, for example the richness or the distinctiveness of encoding in the survival scenario. It is well conceivable that the imagery of different items in a foreign grassland scenario may lead to more diverse processing or bizarre imagery than in a moving scenario. Second, a survival scenario may simply be more exciting and arousing than other contexts, provoking better memory. Given that the fancy vacation scenario in Experiment 1 is also much more exciting than the moving scenario used in Experiment 2 and the original studies, the missing effect on item memory in the first experiment would be easily explained. Third, there may be a valence effect because the survival scenario is inherently threatening, leading to a "threat bias" as compared to more neutral or positive scenarios (De Bruin \& Van Lange, 1999; Peeters \& Czapinski, 1990). This latter effect is itself subject to an evolutionary explanation, and it might be the more fundamental mechanism which operates behind the survival processing advantage. Better memory for details of arousing situations may also be an adaptive feature of memory. Even more, all three (and perhaps more) factors may interact in a particularly efficient manner to produce this fascinating effect.

\section{References}

Anderson, J. R. (1991). Is human cognition adaptive? Behavioral and Brain Sciences, 14, 471-517. doi:10.1017/S0140525X00070801

Barrett, L., Dunbar, R., \& Lycett, J. (2002). Human evolutionary psychology. Princeton, NJ: Princeton University Press.

Bayen, U. J., Murnane, K., \& Erdfelder, E. (1996). Source discrimination, item detection, and multinomial models of source monitoring. Journal of Experimental Psychology: Learning, Memory, and Cognition, 22, 197-215. doi:10.1037/0278-7393.22.1.197

Bröder, A., \& Meiser, T. (2007). Measuring source memory. Zeitschrift für Psychologie/Journal of Psychology, 215, 52-60.

Buss, D. M. (1992). Mate preference mechanisms: Consequences for partner choice and intrasexual competition. In J. H. Barkow, L. Cosmides, \& J. Tooby (Hrsg.), The adapted mind: Evolutionary psychology and the generation of culture. (pp. 249-266). New York, NY: Oxford University Press.

Buss, D. M. (2003). Evolutionary psychology: The new science of the mind (2nd ed.). Boston, MA: Allyn and Bacon.

Butler, A. C., Kang, S. H. K., \& Roediger, H. L. (2009). Congruity effects between materials and processing tasks in the survival processing paradigm. Journal of Experimental Psychology: Learning, Memory, and Cognition, 35, 1477-1486. doi:10.1037/a0017024

Cohen, J. (1988). Statistical power analysis for the social sciences (2nd ed.). Hillsdale: Erlbaum.

Cosmides, L. (1989). The logic of social exchange: Has natural selection shaped how humans reason? Studies with the Wason selection task. Cognition, 31, 187-276. doi:10.1016/0010-0277(89)90023-1

De Bruin, E. N. M., \& Van Lange, P. A. M. (1999). Impression formation and cooperative behavior. European Journal of Social Psychology, 29, 305-328. doi:10.1002/(SICI)1099-0992(199903/05)29:2/3<305::AID-EJSP929 $>3.0 . \mathrm{CO} ; 2-\mathrm{R}$

Eagly, A. H., \& Wood, W. (1999). The origins of sex differences in human behavior: Evolved dispositions versus social roles. American Psychologist, 54, 408-423. doi:10.1037/0003-066X.54.6.408

Flexser, A. J., \& Tulving, E. (1978). Retrieval independence in recognition and recall. Psychological Review, 85, 153-171. doi:10.1037/0033-295X.85.3.153

Godden, D., \& Baddeley, A. (1975). Context-dependent memory in two natural environments: On land and underwater. British Journal of Psychology, 66, 325-331. doi:10.1111/j.2044-8295.1975.tb01468.x

Howe, M. L., \& Derbish, M. H. (2010). On the susceptibility of adaptive memory to false memory illusions. Cognition, 115, 252-267. doi:10.1016/j.cognition.2009.12.016

Johnson, M. K., Hashtroudi, S., \& Lindsay, D. S. (1993). Source monitoring. Psychological Bulletin, 114, 3-28. doi:10.1037/0033-2909.114.1.3

Kang, S. H. K., McDermott, K. B., \& Cohen, S. M. (2008). The mnemonic advantage of processing fitness-relevant information. Memory \& Cognition, 36, 1151-1156. doi:10.3758/MC.36.6.1151

Kroneisen, M. (2010). On the nature of the survival processing effect. Unpublished Dissertation, Mannheim: University of Mannheim.

Kroneisen, M. \& Erdfelder, E. (2011). On the plasticity of the survival processing effect. Journal of Experimental Psychology: Learning, Memory, \& Cognition, 37, 1553-1562. doi:10.1037/a0024493

Meiser, T., \& Bröder, A. (2002). Memory for multidimensional source information. Journal of Experimental Psychology: Learning, Memory, and Cognition, 28, 116-137. doi:10.1037/0278-7393.28.1.116

Murnane, K., \& Bayen, U. J. (1996). An evaluation of empirical measures of source identification. Memory \& Cognition, 24, 417-428. doi:10.3758/BF03200931

Nairne, J. S., \& Pandeirada, J. N. (2008). Adaptive memory: Remembering with a stone-age brain. Current Directions in Psychological Science, 17, 239-243. doi:10.1111/j.1467-8721.2008.00582.x

Nairne, J. S., \& Pandeirada, J. N. S. (2011). Congruity effects in the survival processing paradigm. Journal of Experimental Psychology: Learning, Memory, and Cognition, 37, 539-549. doi: $10.1037 / \mathrm{a} 0021960$

Nairne, J. S., Pandeirada, J. N., \& Thompson, S. R. (2008). Adaptive memory: The comparative value of survival processing. Psychological Science, 19, 176-180. doi:10.1111/j.1467-9280.2008.02064.x

Nairne, J. S., Thompson, S. R., \& Pandeirada, J. N. (2007). Adaptive memory: Survival processing enhances retention. Journal of Experimental Psychology: Learning, Memory, and Cognition, 33, 263-273. doi:10.1037/0278-7393.33.2.263

Nairne, J. S., Pandeirada, J. N., Smith, M. A., Grimaldi, P. J., \& Bauernschmidt, A. (2010). Adaptive memory: Does survival processing enhance nenory for source? 51st Annual Meeting of the Psychonomic Society, St Louis, 18-21 November 2010.

Otgaar, H., \& Smeets, T. (2010). Adaptive memory: Survival processing increases both true and false memory in adults and children. Journal of Experimental Psychology: Learning, Memory, and Cognition, 36, 1010-1016. doi:10.1037/a0019402

Otgaar, H., Smeets, T., \& Van Bergen, S. (2010). Picturing survival memories: Enhanced memory after fitness-relevant processing occurs for verbal and visual stimuli. Memory \& Cognition, 38, 23-28. doi:10.3758/MC.38.1.23

Paivio, A. (1991). Dual coding theory: Retrospect and current status. Canadian Journal of Psychology/Revue canadienne de psychologie, $45,255-287$

Peeters, G., \& Czapinski, J. (1990). Positivenegative asymmetry in evaluations: The distinction between affective and informational negativity effects. In W. Stroebe \& M. Hewstone (Eds.), European Review of Social Psychology. (Vol. 1, pp. 33-60). Chichester: Wiley.

Pinker, S. (1997). How the mind works. New York: Norton \& Co.

Shimamura, A. P., \& Squire, L. R. (1987). A neuropsychological study of fact memory and source amnesia. Journal of Experimental Psychology: Learning, Memory, and Cognition, 13, 464-473. doi: $10.1037 / 0278-7393.13 .3 .464$

Snodgrass, J. G., \& Corwin, J. (1988). Pragmatics of measuring recognition memory: Applications to dementia and amnesia. Journal of Experimental Psychology: General, 117, 34-50. doi:10.1037/0096-3445.117.1.34

Snodgrass, J. G., \& Vanderwart, M. (1980). A standardized set of 260 pictures: Norms for name agreement, image agreement, familiarity, and visual complexity. Journal of Experimental Psychology: Human Learning \& Memory, 6, 174-215. doi:10.1037/0278-7393.6.2.174

Soderstrom, N. C., \& McCabe, D. P. (2011). Are survival processing memory advantages based on ancestral priorities? Psychonomic Bulletin \& Review, 18, 564-569. doi:10.3758/s13423-011-0060-6

Spencer, W. D., \& Raz, N. (1995). Differential effects of aging on memory for content and context: A meta-analysis. Psychology and Aging, 10, 527-539. doi:10.1037/0882-7974.10.4.527

Weinstein, Y., Bugg, J. M., \& Roediger, H. L. (2008). Can the survival recall advantage be explained by basic memory process? Memory \& Cognition, 36, 913-919. doi:10.3758/MC.36.5.913 
Wilkinson, L. \& The Task Force on Statistical Inference (1999). Statistical methods in psychology journals: Guidelines and explanations.

\section{Appendix}

Instructions for participants in Experiment 2 (translated from German):

Survival scenario:

"In this study, I ask you to imagine that you are stranded in the savannah of an unknown country without any vital commodities. During the months to come you must find food and water, and you have to protect yourself from predators.

We will now present a series of words and pictures. You should judge for every item how relevant it would be in such a
American Psychologist, 54, 594-604.

doi:10.1037/0003-066X.54.8.594

situation. Some items may be relevant, others may not be relevant. That is up to your judgment."

Moving scenario:

"In this study, I ask you to imagine that you plan to move to another country into a new house. During the months to come you must find and purchase a new house and transfer your belongings to the new place.

We will now present a series of words and pictures. You should judge for every item how relevant it would be in such a situation. Some items may be relevant, others may not be relevant. That is up to your judgment." 